\title{
BIOLOGÍA Y COMPORTAMIENTO DE Cotesia flavipes Cameron (BRACONIDAE) PARASITOIDE DE Diatraea saccharalis Fabricius (CRAMBIDAE)
}

\author{
BIOLOGY AND BEHAVIOR OF Cotesia flavipes Cameron (BRACONIDAE) \\ PARASITOID OF Diatraea saccharalis Fabricius (CRAMBIDAE)
}

\author{
Sadith Zobeida Astola Mariscal ${ }^{1}$ y Mónica Narrea Cango ${ }^{2}$
}

\begin{abstract}
Resumen
En Piura, Perú, se estudiaron algunos aspectos de la biología y comportamiento de una línea de Cotesia flavipes, procedente de Costa Rica, en larvas de Diatraea saccharalis. El estudio en tres generaciones se realizó en el laboratorio del Centro de Producción de Insectos Útiles (CPIU) de la Empresa Caña Brava, a una temperatura promedio de $28{ }^{\circ} \mathrm{C}$ y $60 \%$ de humedad relativa (HR). Se determinó el ciclo de desarrollo del parasitoide en 15 días: periodo incubación, 2.5 días; estadio larval I, II y III de 3.3; 2.5 y 1.3 días respectivamente; periodo pupal, 5.6 días; proporción de sexos 1:3.9 macho/hembra; periodo de pre-oviposición 37.9 minutos y tiempo de oviposición, 6.1 segundos. La longevidad del adulto es mayor con alimento. El porcentaje de emergencia es de $91.2 \%$ y la hembra parasita 1 a 2 larvas. El apareamiento es polígamo, con reproducción sexual y partenogénica tipo arrenotoquia. El macho copula con 5-14 hembras, el pre-apareamiento oscila entre 1-94 minutos y el tiempo de cópula entre 8-21 segundos. No hay diferencias en el número de larvas parasitadas en los estadios IV, V y VI de $D$. saccharalis.

Palabras clave: parasitoide, Cotesia flavipes, caña de azúcar, biología, comportamiento.
\end{abstract}

\begin{abstract}
Several aspects of the biology and behavior of a Cotesia flavipes line from Costa Rica, and its host Diatraea saccharalis were studied in Piura, Peru. This research was conducted in the Centro de Producción de Insectos Útiles (CPIU) of the Caña Brava Enterprise, at an average temperature of $28{ }^{\circ} \mathrm{C}$ and $60 \%$ humidity. The life cycle of Cotesia flavipes lasts 15 days; the larval period of the first, second and third stages are 3.3; 2.5 and 1.3 days respectively. Pupa lasts 5.6 days and sex ratio $(\mathrm{M}: \mathrm{F})$ is 1:3.9. Adult longevity is greater when it is fed. The pre-oviposition period and oviposition times are 37.9 minutes and 6.1 seconds, respectively. $C$. flavipes females parasitize 1 to 2 larvae. The adult emergency percentage is $91.2 \%$. There are no differences in the number of age-parasitized larvae of Cotesia flavipes C. on stages IV, V and VI of Diatraea saccharalis F. Cotesia flavipes presents polygamous mating, sexual reproduction and parthenogenic reproduction of arrenotochia type. The male copulates from 5-14 females, the pre-mating ranges from 1-94 minutes and the mating time ranges from 8-21 seconds.
\end{abstract}

Key words: parasitoid, Cotesia flavipes, sugarcane, biology, behavior.

\section{Introducción}

El barrenador del tallo, Diatraea saccharalis F. (Lepidoptera: Crambidae), es considerado el insecto plaga, más importante del cultivo de caña de azúcar en el Perú, ya que ocasiona altas pérdidas en todas las zonas donde se siembra dicho cultivo (Vigil, 2012). Aunque actualmente, la principal forma de controlar esta plaga, es a través de liberaciones de la mosca Billaea claripalpis W. (Diptera: Tachinidae), existe inquietud en probar otros controladores como Cotesia flavipes (Hymenoptera: Braconidae). Esta especie no es nueva en el Perú, fue introducida al país en 1975, procedente de Trinidad-Tobago, por gestión de la Central de Cooperativas Agrarias Azucareras; sin embargo, en esa época no se logró establecer y sólo llegó a causar un parasitismo del $18 \%$, lo que motivó la no continuidad de su propagación y liberación (Cueva et al., 1980; Ayquipa, 1984; Risco, 1996). Por su parte, Lva et al. (2010), también reportó el fracaso de este parasitoide como controlador de D. saccharalis en Texas, EEUU, aunque señaló que fueron las condiciones climáticas subtropicales de la zona, las responsables de este fracaso.

Es sabido que las condiciones ambientales influyen no solo sobre el establecimiento de $C$. flavipes, sino también sobre la biología, como lo demuestran los estudios de varios autores (Tabla 1). Contrariamente sobre el comportamiento de esta especie, no hay muchos estudios, sin embargo, es importante resaltar los trabajos de Cueva et al. (1980) y Ayquipa (1984), 
Tabla 1. Estudios de aspectos biológicos realizados con Cotesia flavipes por diferentes autores, indicando la duración y las condiciones ambientales.

\begin{tabular}{|c|c|c|c|c|c|}
\hline Detalle & Días & $\mathbf{T}^{\circ} \mathbf{C}$ & HR \% & Autores & País \\
\hline \multirow[t]{5}{*}{ Periodo de Desarrollo } & 17.2 & 28.0 & 67.9 & Cueva et al. (1980) & Per \\
\hline & 21.6 & 25.3 & 66.3 & Cueva et al. (1980) & Per \\
\hline & 18.5 & 26.8 & 76.0 & Fajardo (1992) & Ecu \\
\hline & 18.2 & 27.0 & 80.0 & Hernández (2010) & Ven \\
\hline & 17.6 & 28.4 & 72.9 & Gómez (2015) & Per \\
\hline \multirow[t]{5}{*}{ Periodo Incubación/Larval } & 11.1 & 28.1 & 67.9 & Cueva et al. (1980) & Per \\
\hline & 10.5 & 25.3 & 66.3 & Cueva et al. (1980) & Per \\
\hline & $10-12$ & 28.0 & 70.0 & Loor (1989) & Ecu \\
\hline & 11.8 & 26.8 & 76.0 & Fajardo (1992) & Ecu \\
\hline & 12.7 & 27.0 & 80.0 & Hernández (2010) & Ven \\
\hline \multirow[t]{5}{*}{ Periodo pupal } & 6.7 & 28.1 & 67.9 & Cueva et al. (1980) & Per \\
\hline & 10.6 & 25.3 & 66.3 & Cueva et al. (1980) & Per \\
\hline & 6.8 & 26.8 & 76.0 & Fajardo (1992) & Ecu \\
\hline & 5.5 & 27.0 & 80.0 & Hernández (2010) & Ven \\
\hline & 4.1 & 28.4 & 72.9 & Gómez (2015) & Per \\
\hline \multirow[t]{3}{*}{ Periodo de incubación } & 3.0 & 26.8 & 76.0 & Fajardo (1992) & Ecu \\
\hline & 3.2 & 27.0 & 80.0 & Hernández (2010) & Ven \\
\hline & 3.7 & 28.4 & 72.9 & Gómez (2015) & Per \\
\hline \multirow[t]{2}{*}{ Periodo Larva I } & 6.2 & 27.0 & 80.0 & Hernández (2010) & Ven \\
\hline & 5.1 & 28.4 & 72.9 & Gómez (2015) & Per \\
\hline \multirow[t]{2}{*}{ Periodo Larva II } & 2.2 & 27.0 & 80.0 & Hernández (2010) & Ven \\
\hline & 2.1 & 28.4 & 72.9 & Gómez (2015) & Per \\
\hline \multirow[t]{2}{*}{ Periodo Larva III } & 1.1 & 27.0 & 80.0 & Hernández (2010) & Ven \\
\hline & 1.2 & 28.4 & 72.9 & Gómez (2015) & Per \\
\hline
\end{tabular}

en el Perú, quienes entre sus muchos aportes al conocimiento de esta especie, nos indican que $C$. flavipes tiene reproducción bisexual y apareamiento polígamo, siendo el macho sexualmente más precoz que la hembra.

Considerando el potencial de $C$. flavipes y sobre todo que en Costa Rica, es la especie que más se utiliza para combatir a D. saccharalis (Badilla, 2002), se realizó el presente trabajo, para determinar la biología, comportamiento y potencial controlador, de una línea de $C$. flavipes procedente de Costa Rica.

\section{Materiales y métodos}

El presente estudio se realizó en las instalaciones del laboratorio del Centro de Producción de Insectos Útiles (CPIU), Fundo Montelima (Empresa Caña Brava), ubicada a latitud $04^{\circ} 51^{\prime} 06.3^{\prime \prime} \mathrm{S}$, longitud $80^{\circ} 55^{\prime} 47.7^{\prime \prime} \mathrm{W}$, provincia Sullana, región Piura, entre noviembre del 2015 y mayo del 2016, registrándose una temperatura promedio de $28^{\circ} \mathrm{C}$ y $60 \%$ de Humedad Relativa (HR), las cuales fueron medidas a través de un termohigrómetro marca "TAYLOR".

Estudio de la biología de $C$. flavipes

Previamente se desarrolló una crianza masal de $D$. saccharalis, con larvas colectadas del mismo fundo y acondicionadas en recipientes de plástico de $37 \mathrm{~mm}$ x $26 \mathrm{~mm}$ x $12 \mathrm{~mm}$, con tapa ventilada. Las larvas fueron alimentadas con trozos de caña de azúcar previamente desinfectados con hipoclorito de sodio de $1 \%$ y mantenidas en un ambiente bajo condiciones no controladas, donde se registró una temperatura promedio de $27.5{ }^{\circ} \mathrm{C}$ y $67.2 \%$ de HR. Al estado de pupa, fueron sexadas y separadas en jaulas, permitiendo la cópula y posterior oviposición en hojas de caña de azúcar; las posturas se evaluaron en forma diaria hasta su emergencia, retirando las larvas del primer estadio a nuevos recipientes y observando el desarrollo de las mismas. La determinación del paso de un estadio al otro, se estableció en función al momento en el que se observó la presencia de la exuvia de la cápsula cefálica, separando las poblaciones en función a su estadio larval y de esta manera, garantizar la disponibilidad de larvas en sus diferentes estadios.

En el estudio de la biología de $C$. flavipes se consideraron las tres primeras generaciones. El parasitoide fue obtenido de un pie de cría en Costa Rica y seleccionados en la etapa de cocón, estos últimos fueron acondicionados en frascos de plástico transparente de $250 \mathrm{~cm}^{3}$, hasta la emergencia de los adultos (228 machos y 114 hembras). Los adultos en grupos de tres (dos machos y una hembra) se colocaron en frascos de plástico transparente de $30 \mathrm{~cm}^{3}$, por 24 horas para la cópula. Al segundo día, en cada frasco se colocó una larva de $D$. saccharalis del V estadio, para que sea parasitada. Inmediatamente después de observar la parasitación (oviposición), se extrajo la larva y se colocó individualmente en una placa de Petri con dieta artificial preparada según la propuesta de Hensley \& Hammond (1968) a base de germen de trigo, levadura y harina de maíz. Estas larvas parasitadas fueron divididas en dos grupos:

Grupo 1 -Sin Disección: Las larvas parasitadas siguieron su desarrollo sin ser manipuladas. La observación fue en forma diaria, con el fin de determinar el periodo incubación/larval (total), desde la oviposición hasta la salida de la larva III del cuerpo del 
hospedero; periodo de prepupa, desde que la larval III sale y forma su cocón y el periodo pupal, desde la formación del cocón hasta la emergencia del adulto. Con estos datos se determinó el periodo de desarrollo.

Grupo 2 - Con Disección: Mediante la técnica de disección (cada 24 horas), se realizó un pequeño corte lateral en los últimos segmentos abdominales de 10 larvas para extraer cuidadosamente los huevos o larvas del parasitoide y registrar su presencia, desarrollo y duración. De esta manera se determinó el periodo incubación y periodo larval. El final del periodo larval se estableció a la salida del hospedero.

Con respecto a las condiciones ambientales, en las tablas 2 y 3 , se indican los datos de la temperatura y humedad relativa registrados para cada etapa de evaluación.

Estudio de Parámetros biológicos y Comportamiento

Los siguientes parámetros se evaluaron en tres generaciones (por cada parámetro se observaron 10 individuos):

El porcentaje de emergencia de adultos, fue obtenido mediante la relación parasitoide emergido/total de cocones. Se evaluaron los cocones producidos por 20 larvas parasitadas. En esta misma población se realizó el estudio de proporción de sexos, mediante la relación macho/hembra. También se determinó el momento y tiempo de emergencia, apareamiento y tipo de reproducción.

La longevidad del adulto, se determinó desde la emergencia hasta la muerte. Para ello cada individuo se colocó en frascos transparentes de $33 \mathrm{~cm}^{3}$ de capacidad, siendo sometidos a los variables alimento y cópula, tanto a machos como a hembras. Así, se estableció lo siguiente: machos y hembras con alimento y sin alimento; mismos grupos que fueron permitidos de copular y no copular. El alimento proporcionado fue a base de agua con azúcar (proporción 1:3).

Adicionalmente a las hembras copuladas con y sin alimento, se les agregó la variable con y sin larva hospedera, exponiendo al grupo de hembras copuladas una larva hospedera para que sea parasitada sólo una vez.

El periodo de pre-oviposición, se estudió en 20 hembras copuladas, a las que se les expuso una larva hospedera, contabilizando este periodo desde la emergencia de la hembra hasta que introduce su ovipositor sobre la larva: Inmediatamente se registró el periodo de oviposición, como el periodo de permanencia del ovipositor dentro de la larva hospedera. La capacidad de parasitismo se estudió en 10 hembras oviplenas y se determinó colocando una hembra adulta copulada dentro de un frasco transparente de $250 \mathrm{~cm}^{3}$ de capacidad con 2, 3, 4, 5 y 6 larvas/envase de $D$. saccharalis registrándose el número de larvas que logró parasitar y el total de parasitoides que emergieron.

Efecto del parasitoidismo, teniendo en cuenta la edad del parasitoide hembra y del estadio larval del hospedero, se evaluó con hembras apareadas de 1, 2, 3 y 4 días de emergidas, y larvas de $D$. saccharalis del IV, V y VI estadio. En frascos de $250 \mathrm{~cm}^{3}$, con tapa de tul fino, se colocó una hembra apareada con tres larvas de D. saccharalis de la misma edad; esto se hizo para cada edad de la hembra y larva hospedera. Después de 24 horas, se independizaron las larvas en placas Petri con dieta artificial, y se observaron en forma diaria para evaluar su desarrollo. Se realizaron 5 repeticiones para cada combinación edad de hembra parasitoide/edad larva hospedera.

Análisis estadístico

Se realizó una comparación de medias entre las tres generaciones en estudio, empleándose el modelo estadístico No paramétrico. La prueba empleada fue la de Kruskal-Wallis, con un nivel de significación de 0.05. Para la comparación de medias de la longevidad de adultos se empleó la prueba estadística de MannWhittman con un nivel de significación de 0.05. Para la comparación de medias del número de larvas parasitadas por edad del parasitoide C. flavipes sobre los estadios larvales IV, V y VI del hospedero $D$. saccharalis se empleó la prueba estadística KruskalWallis, con un nivel de significación de 0.05.

\section{Resultados y discusión}

Periodo de desarrollo de $C$. flavipes

De acuerdo a los resultados, el ciclo de desarrollo fue en promedio 15.0 días (Tabla 2), con diferencias significativas entre la primera generación y las demás este valor fue menor a los obtenidos por otros autores (Tabla 1), que reportan entre 17.2 y 21.6 días, sin embargo, el resultado es bastante cercano al obtenido por Cueva et al. (1980), presumiblemente por la similitud de condiciones ambientales y de la cercanía de las localidades en que se desarrollaron.

Periodo incubación/larval y Periodo pupal

Los 9.4 días en promedio reportados para el periodo incubación/larval también significan el menor dato obtenido para esta etapa de desarrollo en comparación con la de los demás autores (Tabla 2) que reportaron entre 10.5 a 12.7 días, este rango está dentro de lo obtenido en el presente estudio (8-12) e indicaría que no obstante la variación de condiciones ambientales, el valor promedio de esta etapa se mantiene. Caso contrario sucede con el periodo pupal (Tabla 2) ya que el valor obtenido, 5.6 días, si bien es similar a los obtenidos por los demás autores (Tabla 1), difiere sustancialmente con el obtenido por Cueva et al. (1980), a menor temperatura $\left(25^{\circ} \mathrm{C}\right)$ registró casi el doble de lo obtenido en este estudio, lo cual demostraría el efecto de la temperatura sobre este periodo de desarrollo. En el presente estudio se observó que la pupa es exarate y que el cocón blanquecino al inicio de su formación, va tornándose gris oscuro con el paso de los días. 
Tabla 2. Ciclo de desarrollo en las tres generaciones de Cotesia flavipes, bajo condiciones de laboratorio. Sullana, Piura-Perú. 2016.

\begin{tabular}{|c|c|c|c|c|c|c|c|}
\hline \multirow{2}{*}{ G } & \multicolumn{2}{|c|}{ Incubación-Larval } & \multicolumn{2}{|c|}{ Pupa } & \multirow{2}{*}{$\begin{array}{c}\text { Periodo } \\
\text { Desarrollo } \\
\text { Total } \\
\end{array}$} & \multirow{2}{*}{$\begin{array}{c}\text { Promedio } \\
\mathrm{T}\left({ }^{\circ} \mathrm{C}\right)\end{array}$} & \multirow{2}{*}{$\begin{array}{c}\text { Promedio } \\
\text { HR }(\%)\end{array}$} \\
\hline & Prom & Rango & Prom & Rango & & & \\
\hline I & $8.8 \mathrm{a}$ & $8-9$ & $5.3 \mathrm{a}$ & $4-6$ & $14.1 \mathrm{a}$ & $28.9 \pm 0.5$ & $62.1 \pm 2.9$ \\
\hline II & $9.5 \mathrm{~b}$ & $9-12$ & $5.8 \mathrm{~b}$ & $5-6$ & $15.3 b$ & $28.5 \pm 0.5$ & $60.6 \pm 2.9$ \\
\hline \multirow[t]{2}{*}{ III } & $9.9 \mathrm{~b}$ & $9-12$ & $5.7 b$ & $5-6$ & $15.6 b$ & $28.0 \pm 0.2$ & $58.0 \pm 1.6$ \\
\hline & 9.4 & 8-12 & 5.6 & 4-6 & 15.0 & $28.5 \pm 0.4$ & $60.2 \pm 2.5$ \\
\hline
\end{tabular}

En cuanto a las generaciones, se observó diferencias significativas entre la primera con la segunda y tercera generación.

\section{Huevos - Periodo de incubación}

Los huevos, visibles en las larvas a las que se hizo la disección, se observaron traslúcidos y de forma ovalada con ambos extremos ligeramente redondeados; pero uno más angosto que el otro (Figura 1). Gracias al procedimiento de disección fue posible evidenciar la forma y color, y además determinar con precisión el periodo de incubación, el cual fue en promedio 2.5 días (Tabla 3). Este valor fue también ligeramente inferior, pero bastante cercano a los obtenidos por otros autores (Tabla 1), bajo condiciones ambientales muy diferentes a las del presente estudio, lo que demostraría que al desarrollarse los huevos dentro de la larva del hospedero, las condiciones ambientales no influyen en su Periodo de incubación. En este estudio también se encontraron diferencias significativas entre las tres generaciones, específicamente de la segunda con la primera y tercera generación.

Tabla 3. Periodo de Incubación y periodo larval de Cotesia flavipes, bajo condiciones de laboratorio. Sullana, Piura-Perú. 2016.

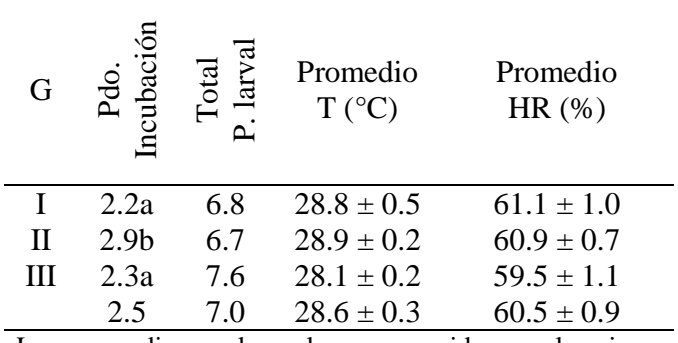

Los promedios en las columnas seguidas por la misma letra no difieren estadísticamente $(\alpha=0.05)$.

\section{Larvas -Periodo larval}

Nuestro estudio, demostró la diferencia de tres estadios larvales en $C$. flavipes, los cuales se desarrollaron en el interior de la larva hospedera, emergiendo al termino del tercero, con ayuda de sus mandíbulas, que perforan el tegumento, sin preferencia alguna en cualquier parte del cuerpo de la larva hospedera; iniciando de inmediato la formación del del cocón. La larva I (Figura 2), es algo aplanada, móvil y traslúcida, que permite ver su tubo digestivo a lo largo de todo el cuerpo; presenta en la parte posterior del cuerpo una leve constricción muy característica. La Larva II (Figura 3), cilíndrica, crema con líneas intersegmentarias marcadas, tiene el último segmento en forma de vesícula y es más estrecha que la larva I. La larva III (Figura 4), es la menos móvil, con la cápsula cefálica esclerotizada muy visible y la parte posterior del cuerpo mucho más estrecha que la larva II, tomando una forma de globo.

Parámetros biológicos

Emergencia de adultos: Los resultados indican un porcentaje de emergencia de $89.8 ; 87.1$ y $96.8 \%$ para las generaciones I, II y III respectivamente, lo que evidencia un dato prometedor sobre esta línea del parasitoide. De todos estos adultos emergidos, el $40 \%$ lo hizo entre las 00:00-06:00 horas, 30\% entre las 06:00-12:00 horas y 30\% entre las 12:00-00:00 horas.

Proporción de sexos: El número de individuos hembras fue mayor en las tres generaciones, dando una relación promedio de 1:3.9, resultado muy similar al encontrado por Fajardo (1992), quien establece una relación promedio 1:4. Los adultos se reconocen por la longitud de sus antenas (Figura 5).

Longevidad -efecto de la alimentación, cópula y oviposición: Los resultados de la longevidad mostraron el efecto de las variables alimento, cópula y oviposición, siendo el factor alimento el más relevante sobre la vida de $C$. flavipes. En general los individuos que se alimentaron (tanto machos como hembras) vivieron más que aquellos que no se alimentaron (Figura 5).

En cuanto a los machos que se alimentaron, no hubo diferencias significativas entre la longevidad de los que copularon (3.59 días) y no copularon (4.0 días), pero si se encontraron diferencias cuando estos no se alimentaron ( 0.75 días cuando copularon y 1.68 cuando no copularon). En ambos casos, alimentados o no, aquellos que no copularon vivieron más que los que si copularon, presumiblemente el esfuerzo y desgaste por copular, afecta su longevidad.

Las hembras con alimento, cópula y larva hospedera tuvieron una longevidad promedio de 2.91 días, mientras que las que no tuvieron presencia de larva hospedera vivieron 3.89 días. Por su parte las hembras que no recibieron alimento, copularon $\mathrm{y}$ fueron expuestas a la larva hospedera vivieron 0.96 días y las que no, 1.56 días, en todos estos casos si hubo 


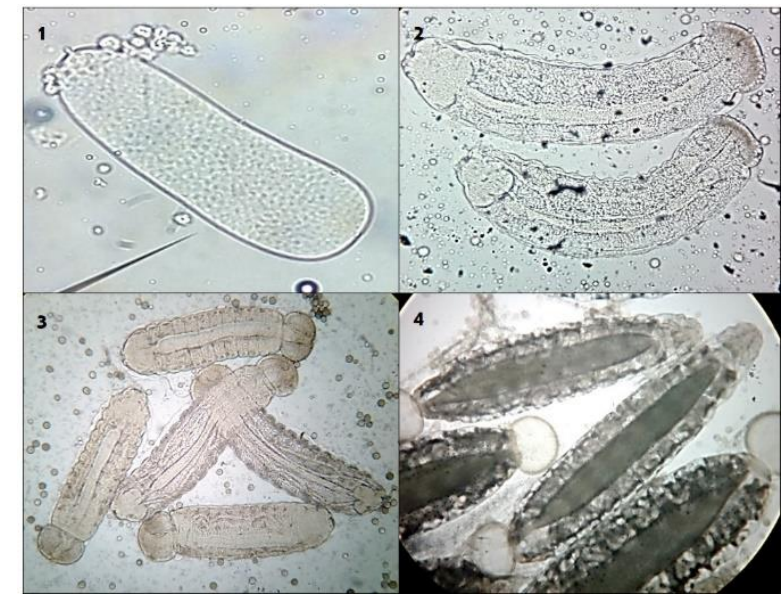

Figuras 1-4. Cotesia flavipes. 1) Huevo. 2) Primer estadio larval. 3) Segundo estadio larval. 4) Tercer estadio larval (Foto: Astola, 2016).

diferencias significativas y al igual que en el caso de los machos, alimentadas o no, las hembras que no fueron expuestas a larva hospedera vivieron más días que las que sí lo hicieron. En cuanto a que aquellas hembras que no copularon, fue evidente el factor alimento en la duración de la longevidad.

Los resultados confirman lo mencionado por Jervis \& Copland (1996), la longevidad de los parasitoides está influenciada por diversos factores tales como tamaño del insecto, apareamiento, oviposición, temperatura, humedad, fotoperiodo y dieta. Carey et al. (1998), señalaron que los insectos que son sometidos a apareamiento y parasitación tienen menos tiempo de vida que los individuos vírgenes, esto debido al costo de energía de la reproducción.

Periodo de pre-oviposición y tiempo de oviposición: La pre-oviposición fue breve, apenas 37.9 minutos, con un rango de 15 a 78 minutos, dato superior al reportado por Cueva et al. (1980) quienes registraron apenas 6.8 segundos. En cuanto al tiempo que les toma oviponer registramos un tiempo de 6.1 segundos, con un rango de 3.8 a 8.8 segundos. Los datos son

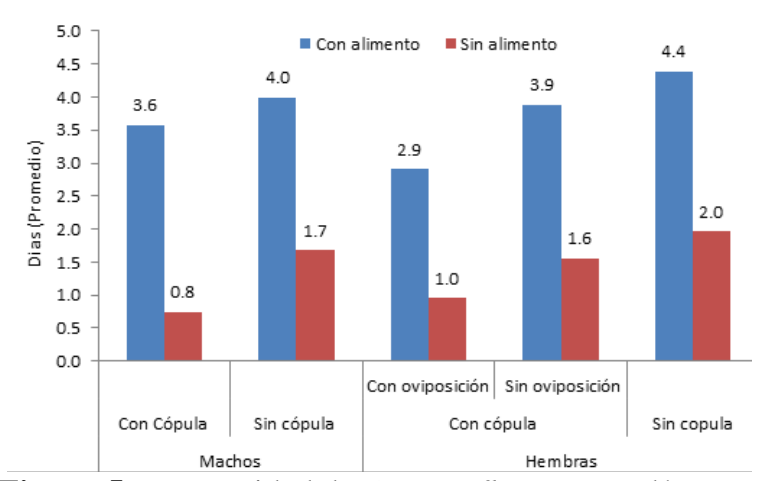

Figura 5. Longevidad de Cotesia flavipes, en días, por efecto de la alimentación, cópula y oviposición, bajo condiciones de laboratorio. Sullana-Piura. 2016.

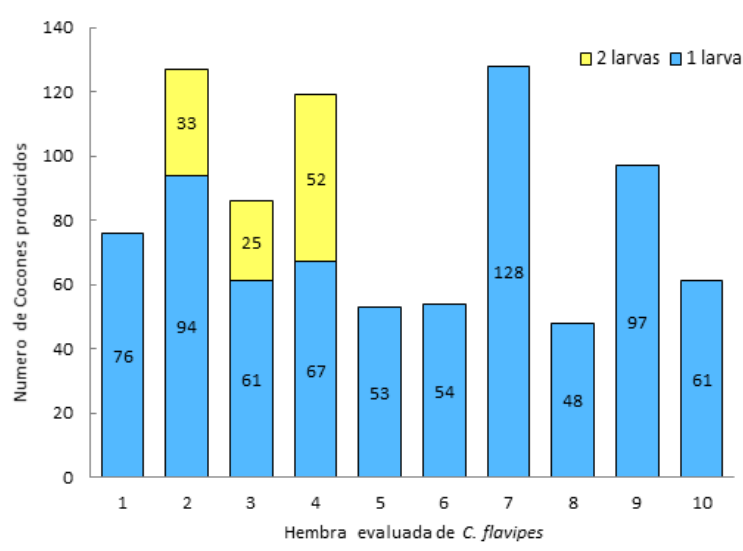

Figura 6. Capacidad de parasitismo de Cotesia flavipes sobre larvas de Diatraea saccharalis, bajo condiciones de laboratorio. Sullana, Piura-Perú. 2016.

coherentes con la biología de un insecto parasitoide de corta vida, como $C$. flavipes.

\section{Comportamiento de $C$. flavipes}

Emergencia del adulto: El adulto de C. flavipes, inicia su salida masticando el cocón en un solo extremo (donde se encuentra su cabeza), emerge primero la cabeza y luego las patas anteriores, que usa para impulsarse y con ayuda de las alas prosigue a sacar sus patas posteriores y el resto de su cuerpo. Al término de la emergencia camina unos segundos y luego limpia su cuerpo con su aparato bucal. Este comportamiento no difiere a lo que señalan Cueva et al. (1980) y Gómez (2015).

Reproducción: Corroborando lo descrito por Cueva et al. (1980), Ayquipa (1984) y Fajardo (1992), se observó que $C$. flavipes, presenta reproducción sexual (produce machos y hembras) y partenogénica tipo arrenotoquia (sólo produce machos).

Apareamiento y Cópula: Inmediatamente de emergido, el macho empieza su comportamiento de apareamiento, primero extiende las alas y las agita rápidamente sin volar y de ésta manera se acerca a la hembra por atrás, si la hembra acepta el cortejo se queda inmóvil; en seguida él se coloca sobre ella y doblando su abdomen introduce su genital, para luego extender sus alas. Por unos 8-21 segundos ambos quedan inmóviles, lo que se interpreta como tiempo de cópula. La hembra inicia la separación agitando sus alas para liberarse del macho, luego este hace un pequeño salto hacia atrás y se separan. En total todo el apareamiento dura de 1-94 minutos, con un promedio de 32.6 minutos. Ambos son polígamos y pueden copular con otros individuos, el macho hasta con 5 - 14 hembras. Aunque nuestros valores difieren con los obtenidos por Cueva et al. (1980) y Gómez (2015), el comportamiento observado fue el mismo.

Oviposición: Las hembras inmediatamente copuladas y en presencia de larvas de D. saccharalis proceden a ovipositarlas. Primero caminan alrededor y sobre la larva, hasta escoger el lugar, donde insertaran 


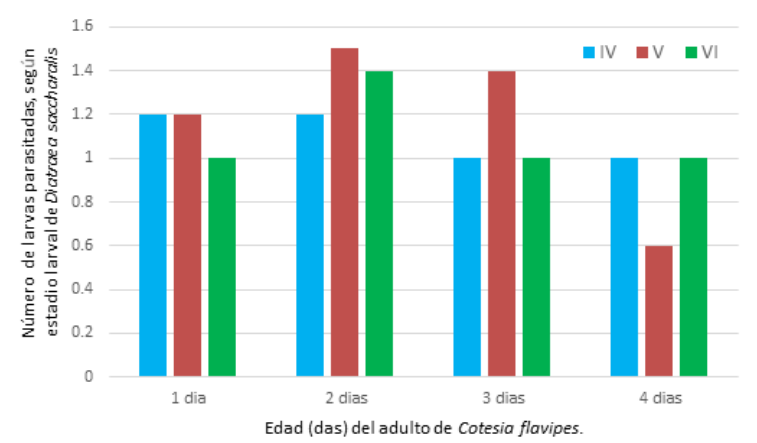

Figura 7. Número de larvas parasitadas de Diatraea saccharalis, según estadio larval por el adulto de Cotesia flavipes, según su edad (días), bajo condiciones de laboratorio. Sullana, Piura-Perú. 2016.

su ovipositor, generalmente en los últimos segmentos abdominales, luego extienden las alas, penetran a la larva y se retira inmediatamente. La larva hospedera generalmente reacciona ya sea contorsionado su cuerpo o vomitando un líquido, que si cae sobre el parasitoide, lo inmoviliza temporalmente. En ocasiones la larva, logra morder al parasitoide, evitando la parasitoidismo.

Capacidad de parasitismo: Encontramos en 10 hembras que 7 ovipositaron sólo en una larva hospedera, los 3 restantes ovipositaron en dos larvas. En este último caso las segundas larvas parasitadas produjeron menos cocones que las primeras, sin embargo, la suma total de cocones producidos fue mayor al promedio de las demás (Figura 6).

Número de larvas parasitadas por edad: Las hembras de dos días de edad parasitaron más que las hembras de cuatro días, pero en términos generales no se evidenció una influencia de la edad (en días) del parasitoide $C$. flavipes, sobre la preferencia al parasitar cualquiera de los últimos tres estadios larvales de su hospedero (Figura 7). En todas las evaluaciones se encontró que la parasitación ocurría entre 1 a 2 larvas hospederas, corroborando los resultados anteriores.

\section{Conclusiones}

La línea de $C$. flavipes, procedente de Costa Rica e introducida en Piura-Perú tiene una biología y comportamiento similar a otras líneas del parasitoide, que se desarrollan en otras zonas azucares del Perú y del Mundo.

El ciclo de desarrollo de la línea costarricense de $C$. flavipes, fue en promedio 15 días. La emergencia de los adultos se realiza en horas de la madrugada y de la mañana y aunque su longevidad es corta (5 días), son más longevos cuando se alimentan.

La línea costarricense de $C$. flavipes no tiene preferencia para parasitar los estadios larvales IV, V y VI de $D$. saccharalis lo que indica su alto valor como parasitoide, pues puede liberarse en cualquier momento que se detecte la plaga.

La línea costarricense de $C$. flavipes tiene apareamiento polígamo. El macho copula con 5 a 14 hembras. El promedio de pre-apareamiento fue de 32.6 minutos y el tiempo promedio de cópula fue 13.4 segundos, además presenta reproducción sexual y partenogénesis tipo arrenotoquia.

Se demuestra el alto valor de esta línea del parasitoide como controlador de D. saccharalis, debido a su corto ciclo de desarrollo, reproducción y su comportamiento agresivo, factores que sin duda demuestran la necesidad de continuar su crianza y liberación en los campos de caña de azúcar.

\section{Agradecimientos}

A la empresa Caña Brava por el financiamiento y por facilitar los materiales e instalaciones de su laboratorio para el desarrollo de la investigación. A la Ing. Gaby Cárdenas por su apoyo durante el desarrollo de la investigación.

\section{Literatura citada}

Ayquipa G. 1984. Crianza masiva y aspectos de la biología de Apanteles flavipes Cameron (Hym.: Braconidae) en larvas de Diatraea saccharalis Fabr. (Lep.: Crambidae). Tesis Ph D. Trujillo, PE. UNT.

Badilla F. 2002. Un programa exitoso de control biológico de insectos plaga de la caña de azúcar en Costa Rica. Manejo Integrado de Plagas y agroecología, 64: 77-87.

Carey J., Liedo P., Müller H., Wang J. \& Chiou J. 1998. Relationship of age patterns of fecundity to mortality, longevity, and lifetime reproduction in a large cohort of Mediterranean fruit fly females. Journal of Gerontology, 53: 245-251.

Cueva M., Ayquipa G. \& Mescua V. 1980. Estudios sobre Apanteles flavipes (Cameron) introducidos para controlar Diatraea saccharalis (F.) en el Perú. Revista Peruana de Entomología, 23(1): 73-76.

Fajardo J. 1992. Biología, multiplicación y liberación de Cotesia (=Apanteles) flavipes para el control del barrenador del tallo, Diatraea saccharalis en maíz. Tesis Ing. Agrónomo. Los Ríos, Ecuador. Universidad Técnica de Babahoyo.

Gómez G. 2015. Ciclo biológico y comportamiento de Cotesia (Apanteles) flavipes, en larvas de Diatraea saccharalis Fabricius Casa Grande, La Libertad - Perú. 2014. Tesis de grado- Universidad Nacional de Trujillo.

Hensley S. \& Hammond A. Jr. 1968. Laboratory techniques for rearing the sugar cane borer on an artificial diet. Journal of Economic Entomology, 61(6): 1742-1743. DOI: https://doi.org/10.1093/jee/61.6.1742.

Hernández D. 2010. Estudio de algunos aspectos biológicos de Cotesia flavipes Cameron (Hymenoptera: Braconidae) parasitoide de Diatraea saccharalis Fabricius (Lepidoptera: Crambidae). Entomotrópica, 25(2): 69-81.

Jervis M. \& Copland M. 1996. The life cycle. In: Jervis M. \& Kidd N. (eds.). Insect natural enemies: practical approaches to their study and evaluation. . 63-161. Chapman \& Hall, London.

Loor H. 1989. Introducción y establecimiento de Cotesia flavipes Cameron (Hymenoptera: Braconidae) para el control biológico de Diatraea spp. Tesis de Ing. Agr. Guayaquil, Ecuador. UG. 
Lva J., Wilsona L., Beuzelim J., White W. \& Reagan T. 2010. Impact of Cotesia flavipes (Hymenoptera: Braconidae) as an augmentative biocontrol agent for the sugarcane borer (Lepidoptera: Crambidae) on rice. Biological Control, 56(2): 159-169.

Risco S. 1996. Éxitos históricos de taquínidos y bracónidos en el control biológico de Diatraea en caña de azúcar cultivada en América. Rev. Per. Ent., 39: 85-90.
Vigil D. 2012. Principales plagas y enfermedades en el cultivo de caña de azúcar. Guía Técnica: Manejo Integrado de Plagas en el cultivo de caña de azúcar. Oficina Académica de Extensión y Proyección Social de la Universidad Nacional Agraria La Molina, 1(1): 7-19.

\footnotetext{
${ }^{1}$ Departamento de Entomología -Facultad de Agronomía Universidad Nacional Agraria La Molina Apartado Postal 12-056. Lima 12 - Perú.

2 Departamento de Entomología -Facultad de Agronomía Universidad Nacional Agraria La Molina Apartado Postal 12-056. Lima 12 - Perú. Autor de correspondencia: mnarrea@lamolina.edu.pe.
} 